\title{
Estimação de parâmetros cinéticos da reação de cura do sistema DGEBA - mPDA
}

Kinetic parameter estimation of the curing reaction of the system DGEBA-mPDA

\author{
L. S. Andrade ${ }^{1}$; W. S. Oliveira ${ }^{1}$; W. A. C. Lima' ; P. L. Santana ${ }^{2}$; V. M. A.Calado ${ }^{3}$; \\ R. L. Pagano ${ }^{2 *}$

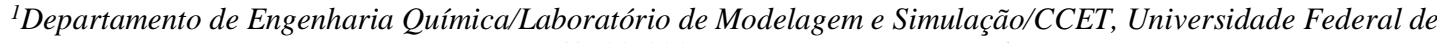 \\ Sergipe, 49100-000, São Cristóvão/SE,Brasil \\ ${ }^{2}$ Programa de Pós-graduação em Engenharia Química/PEQ, Universidade Federal de Sergipe, 49100-000, São \\ Cristóvão/SE,Brasil \\ ${ }^{3}$ Universidade Federal do Rio de Janeiro, Escola de Química, Rio de Janeiro/RJ, Brasil \\ *rpagano@ufs.br \\ (Recebido em 25 de julho de 2017; aceito em 24 de maio de 2018)
}

\begin{abstract}
A estimação de parâmetros é um campo bastante importante na área de engenharia, visto sua relação com o desenvolvimento de modelos matemáticos capazes de representar situações práticas como o projeto, a otimização e o controle dos processos químicos. A reação de cura para produção de materiais compósitos, caracterizada pela grande liberação de energia em moderadas e altas temperaturas, configura-se como um desses processos. Este estudo objetivou a determinação de parâmetros cinéticos de quatro modelos consagrados na literatura, sendo dois mecanicistas e dois empíricos, a partir de dados experimentais isotérmicos e não isotérmicos da reação da resina epóxi diglicidil éter de bisfenol-A (DGEBA) com o agente endurecedor m-fenileno diamina (m-PDA). As condições isotérmicas adotadas foram nas temperaturas de $354,5 \mathrm{~K}, 364,5 \mathrm{~K}, 374,5 \mathrm{~K}$ e $384,5 \mathrm{~K}$, já as não isotérmicas utilizaram taxas de aquecimento de $2,5 \mathrm{~K} \mathrm{~s}^{-1}, 5$ $\mathrm{K} \mathrm{s}^{-1}$ e $10 \mathrm{~K} \mathrm{~s}^{-1}$, aplicando a técnica de DSC (Calorimetria diferencial de varredura). Os modelos foram solucionados pela sub-rotina DASSL, estimando os parâmetros por meio do programa ESTIMA, o qual consiste no algoritmo determinístico de Gauss-Newton para encontrar o valor mínimo da função objetivo de mínimos quadrados e no algoritmo heurístico Particle Swarm Optimization (PSO) para estimação dos parâmetros dos modelos propriamente dita. Os valores dos parâmetros mostraram-se em concordância com as faixas comumente relatadas na literatura para todos os modelos. O Modelo de Horie foi o que apresentou resultados mais satisfatórios, principalmente, devido à sua simplicidade e o menor número de parâmetros que descrevem a cinética da reação.

Palavras-chave: estimação de parâmetros, cinética de cura, DSC.
\end{abstract}

Parameter estimation is a very important field in engineering area, since its relation with the development of mathematical models able to represent practical situations as the design, the optimization and the control of chemical processes. The curing reaction for the production of composite materials, characterized by the large release of energy in moderate and high temperatures, is configured as one of these processes. This study aimed to determinate the kinetic parameters of four established models in the literature, two of them mechanistic and two empiric, from isothermal and non-isothermal experimental data of the reaction between epoxy resin diglycidyl ether of bisphenol-A with hardener m-phenylene diamine (m-PDA) at temperatures $354,5 \mathrm{~K}, 364,5 \mathrm{~K}, 374,5 \mathrm{~K}$ and $384,5 \mathrm{~K}$ and at heating rates of $2,5 \mathrm{~K} \mathrm{~s}^{-1}, 5 \mathrm{~K} \mathrm{~s}^{-1}$ and $10 \mathrm{~K} \mathrm{~s}^{-1}$, obtained by the DSC technique (Differential scanning calorimetry). The models were solved by the subroutine DASSL, estimating the parameters through ESTIMA software which consists of the deterministic Gauss-Newton algorithm to find the minimum value of the least squares objective function and heuristic PSO (Particle Swarm Optimization) algorithm for parameter estimation. The values of the parameters are in agreement to the ranges commonly reported in the literature for all the models. The model of Horie presented more satisfactory results, especially due to simplicity and the lower number of parameters that describe the kinetic of the reaction.

Keywords: parameter estimation, cure kinetic, DSC. 


\section{INTRODUÇÃO}

Os materiais compósitos são classificados, de forma geral, como uma família relativamente nova de materiais caracterizados por melhores propriedades do que os convencionais. A combinação de dois ou mais materiais em uma escala macroscópica resultam em um terceiro, macroscopicamente homogêneo, que apresenta características inerentes aos materiais formadores ou até mesmo qualidades distintas dos seus precursores.

Dentre as propriedades que podem ser melhoradas, em um compósito polimérico, podem ser citadas a diminuição do peso molecular e o aumento da resistência. No entanto, qualidades como rigidez, resistência à corrosão, aumento da vida útil, condutividade térmica ou isolamento acústico são requeridas a depender do fim a que o material se destina.

Existem vários tipos de compósitos dentre os quais se destacam os compósitos metálicos, poliméricos e cerâmicos [1]. Os compósitos poliméricos são constituídos por resinas poliméricas geralmente de baixa resistência e rigidez (matriz ou fase contínua) e por elementos de reforço.

O comportamento das resinas perante a temperatura leva a classificá-las como termorrígidas ou termoplásticas. Os termoplásticos são polímeros que apresentam moléculas constituídas de cadeias predominantemente lineares, sujeitas a interações secundárias entre moléculas adjacentes. Depois de aquecido, esse polímero pode deformar, amolecer ou plastificar, possibilitando moldagem e, em seguida, resfriando e endurecendo, reversivelmente até níveis de temperatura abaixo de sua temperatura de degradação [2]. O PET (politereftalato de etileno) e o PVC (policloreto de vinila) são exemplos de resinas termoplásticas.

As resinas termorrígidas ou termofixas apresentam ligações cruzadas intermoleculares, resultando em uma estrutura reticulada (chamada de rede), infusível e insolúvel, que se alteram quimicamente sob aquecimento. As uniões entre as cadeias resultam de reações de reticulação (reações de cura). A preparação e as estruturas químicas destas resinas influenciam o mecanismo de cura e afetam diretamente a qualidade do produto final [2]. As resinas fenólicas, melamínicas e epoxídicas são as mais utilizadas atualmente.

As resinas epóxi, descobertas em 1909 pelo químico russo Prilechajew são definidas como polímeros de baixo peso molecular que contém um ou mais grupos epoxídicos como mostra a Figura $1[3]$.

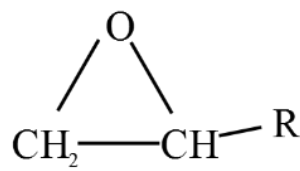

Figura 1: Grupo funcional epóxi.

O fato das resinas epóxi serem os polímeros termorrígidos mais utilizados deve-se às suas propriedades como ótima resistência química, estabilidade térmica e grande capacidade de aderência [4]. Estas propriedades dependem da combinação específica entre o tipo de resina epóxi e do agente reticulante utilizados durante a reação de polimerização, possibilitando aplicá-las em diversos campos, dentre os quais podem-se citar revestimentos, adesivos, componentes elétricos, pisos industriais, tubulações e tanques de armazenamento.

A investigação do processo de cura das resinas epóxi para melhor controle das propriedades finais dos compósitos que atendam com qualidade a aplicação para a qual foram designados tornase, portanto, de grande significância e o objetivo geral deste estudo, já que tais propriedades são determinadas principalmente pela composição química e pelas condições nas quais a reação de cura ocorre.

As resinas epoxídicas são resinas termorrígidas que são curadas utilizando-se larga variedade de agentes de cura ou reticulantes via as chamadas reações de cura. Dentre os vários tipos, destacam-se as resinas epóxi à base do Bisfenol-A e de produtos naturais tais como carboidratos, proteínas, gorduras e óleos [3].

O diglicidil éter de bisfenol-A (DGEBA) é produzido pela reação da epicloridrina com o bisfenol-A na presença de um catalisador básico. A Figura 2 mostra a estrutura química do DGEBA. As propriedades da resina epoxídica DGEBA dependem do número de unidades que se repetem. Moléculas de baixo peso molecular são geralmente líquidas, enquanto as moléculas de alto peso molecular tendem a ser líquidos mais viscosos ou sólidos [3]. A importância deste composto é 
notável na indústria química, visto que $75 \%$ das resinas epóxi produzidas no mundo todo são baseadas no DGEBA [5].

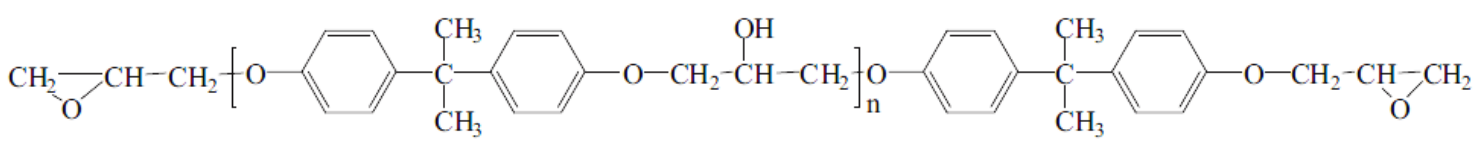

Figura 2: Estrutura química do DGEBA.

Uma variedade de reagentes auxiliares pode ser utilizado para curar resinas epoxídicas através do processo de polimerização. Esses reagentes incluem as aminas, ácidos, isocianatos e mercaptanos [6]. As aminas são as mais utilizadas e são aplicadas em diferentes propósitos a depender da sua estrutura. Aminas alifáticas são muito reativas e possibilitam a cura mesmo à temperatura ambiente. A principal vantagem das aminas cicloalifáticas é sua versatilidade: elas demonstram bom equilíbrio entre a reatividade e as propriedades finais. As aminas aromáticas, por sua vez, são menos reativas, mas fornecem boa resistência térmica e química [5].

Como exemplo de amina aromática, pode-se citar a meta-fenilenodiamina (m-PDA) que foi utilizada como agente endurecedor do DGEBA neste trabalho. Zvetkov (2001) [7] e Pagano et al. (2008) [8] também estudaram a reação entre o DGEBA e o m-PDA. A molécula do m-PDA é apresentada na Figura 3.

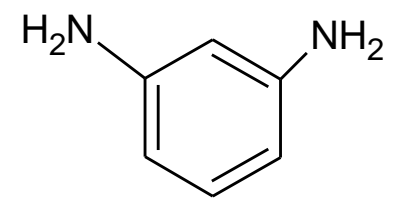

Figura 3: Estrutura química do m-PDA.

As reações de cura são altamente exotérmicas em moderadas e altas temperaturas ocasionando gradientes significativos. Portanto, o processamento dessas resinas requer conhecimento substancial da cinética da reação [7]. Dados da cinética de cura fornecem informações para otimização dos ciclos de cura que reduzem o custo de produção ou melhoram as propriedades dos produtos [2].

Os métodos utilizados para estudar a cinética de cura podem ser classificados em mecanicistas ou empíricos. A abordagem mecanicista deriva do balanço das espécies que participam da reação. Geralmente, esta abordagem torna-se complexa devido às interações entre a cinética química e as mudanças das propriedades físicas do material, mas possui como vantagem o fato de não depender das condições da reação, além de possuir notável capacidade de previsão da reação. A abordagem empírica, na qual equações de taxa são desenvolvidas a partir da regressão dos dados cinéticos, por sua vez, apresenta uma maior simplicidade e menor custo computacional, o que lhe confere maior aceitação industrial. No entanto, esta abordagem não é recomendada para predições fora da faixa original dos dados experimentais [9-10].

De acordo com Zvetkov (2002) [11], o mecanismo de cura epóxi-amina é geralmente descrito pelo esquema proposto por Horie et al. (1970) [12] o qual é apresentado a seguir pelas reações 1(a)$1(\mathrm{~d})$ :

sendo:

$$
\begin{aligned}
& \mathrm{Ep}+\mathrm{RNH}_{2}+\mathrm{EpOH} \underset{\mathrm{k}_{1}^{\prime}}{\stackrel{\mathrm{k}_{1}}{\rightarrow}} \mathrm{RR} \mathrm{R}^{\prime} \mathrm{NH}+\mathrm{EpOH} \\
& \mathrm{Ep}+\mathrm{RNH}_{2}+\mathrm{R}_{\mathrm{i}} \mathrm{OH} \stackrel{\underset{\mathrm{k}_{2}}{\rightarrow}}{\mathrm{RR}} \mathrm{RH}^{\prime}+\mathrm{R}_{\mathrm{i}} \mathrm{OH} \\
& \mathrm{Ep}+\mathrm{RR}^{\prime} \mathrm{NH}+\mathrm{EpOH} \stackrel{\mathrm{k}_{2}}{\rightarrow} \mathrm{RR}^{\prime} \mathrm{R}^{\prime} \mathrm{N}+\mathrm{EpOH} \\
& \mathrm{Ep}+\mathrm{RR}^{\prime} \mathrm{NH}+\mathrm{R}_{\mathrm{i}} \mathrm{OH} \stackrel{\mathrm{k}_{2}^{\prime}}{\rightarrow} \mathrm{RR}^{\prime} \mathrm{R}^{\prime \prime} \mathrm{N}+\mathrm{R}_{\mathrm{i}} \mathrm{OH}
\end{aligned}
$$

Ep - epóxi;

$\mathrm{RNH}_{2}, \mathrm{RR}$ 'NH, RR'R"N - aminas primária, secundária e terciária, respectivamente; 
EpOH - espécie acumulada durante a reação contendo grupos hidroxilas catalisadores; $\mathrm{R}_{\mathrm{i}} \mathrm{OH}$ - impurezas contendo grupos hidroxilas catalisadores.

Os termos R' e R" representam radicais oriundos da resina epóxi, enquanto as constantes de velocidade de Arrhenius são representadas pela letra " $k$ ", sendo $k_{1}$ e $k_{2}$ as constantes referentes ao caminho autocatalítico da reação e $\mathrm{k}_{1}{ }^{\prime}$ e $\mathrm{k}_{2}$ ' as constantes que se referem ao caminho não catalítico do mecanismo descrito.

Para a análise das reações de cura, acompanha-se o grau de conversão da reação utilizando-se diferentes métodos físico-químicos para análise dos grupos envolvidos na reação, como a técnica de DSC (Calorimetria diferencial de varredura) [13]. Esta técnica de DSC possui a vantagem de ser o único método que permite medir com boa precisão tanto a taxa de reação quanto o grau de conversão. Portanto, a técnica fornece os valores das variáveis requeridas para solução das equações de transferência de calor e massa: o fluxo de calor (proporcional à taxa de reação) e o calor gerado (proporcional ao grau de conversão). A técnica de DSC pode ser conduzida tanto no modo isotérmico, no qual o sistema mantém-se em uma temperatura constante, quanto no modo não isotérmico, no qual a amostra é aquecida a uma taxa constante até que a reação se processe completamente.

A hipótese básica para aplicação da técnica de DSC para a cura de resinas termorrígidas é de que a taxa de reação é considerada diretamente proporcional ao fluxo de calor e pode ser matematicamente expressa pela Equação (2) [14-15]:

$$
\frac{d \alpha}{d t}=\frac{1}{\Delta H_{T}}\left(\frac{d \Delta H}{d t}\right)=k f(\alpha)
$$

O grau de conversão $\alpha$ é obtido, portanto, a partir da Equação (3):

$$
\alpha=\frac{\Delta H}{\Delta H_{T}}
$$

em que $\Delta H$ representa o calor liberado pela reação em um dado tempo $t, \Delta H_{T}$ o calor total liberado pela reação, " $k$ " é uma constante cinética a uma dada temperatura $T$ e $f(\alpha)$ representa um modelo capaz de predizer o comportamento do sistema reacional.

O presente trabalho objetivou estimar parâmetros cinéticos da reação de cura de uma matriz epóxi com um agente reticulante, tanto no regime isotérmico quanto no regime não isotérmico, com diferentes taxas de aquecimento no equipamento DSC (Differential Scanning Calorimeter), considerando modelos empíricos e mecanicistas.

\section{MATERIAL E MÉTODOS}

Os dados isotérmicos nas temperaturas de $354,5 \mathrm{~K}, 364,5 \mathrm{~K}, 374,5 \mathrm{~K}$ e $384,5 \mathrm{~K}$ e não isotérmicos da reação entre a resina epoxídica DGEBA e o agente reticulante m-PDA em três taxas de aquecimento distintas $\left(2,5 \mathrm{~K} \mathrm{~s}^{-1}, 5 \mathrm{~K} \mathrm{~s}^{-1} \mathrm{e} 10 \mathrm{~K} \mathrm{~s}^{-1}\right)$ foram ajustados a quatro diferentes modelos reportados na literatura, com o objetivo de estimar os parâmetros que melhor descrevem o comportamento experimental. Os modelos utilizados são descritos pelas Equações 4, 5, 6 e 7.

Estes modelos foram solucionados pela sub-rotina DASSL, estimando os parâmetros por meio do programa ESTIMA. Entre as aplicações do ESTIMA, está na estimação de parâmetros cinéticos de um meio reacional, como no processo de produção de etanol utilizando levedura [18]. Este programa consiste no algoritmo determinístico de Gauss-Newton para encontrar o valor mínimo da função objetivo de mínimos quadrados e no algoritmo heurístico Particle Swarm Optimization (PSO) para estimação dos parâmetros dos modelos.

- Modelo 1: Modelo de Horie et al. [12]

$$
\frac{d \alpha}{d t}=K_{1}(B+\alpha)(1-\alpha)^{2}
$$


- Modelo 2: Modelo de Kamal et al. [16]

$$
\frac{d \alpha}{d t}=K_{1}\left(B+\alpha^{m}\right)(1-\alpha)^{n}
$$

- Modelo 3: Modelo da razão de reatividade [11]

$$
\begin{aligned}
\frac{d \alpha}{d t} & =K_{1}(B+\alpha)(1-\alpha)\left(r \lambda_{1}+\lambda_{0}\right) \\
-\frac{d \lambda_{1}}{d t} & =K_{1}(B+\alpha)(1-\alpha)\left(r \lambda_{1}-\lambda_{0}\right) \\
-\frac{d \lambda_{0}}{d t} & =2 K_{1}(B+\alpha)(1-\alpha) \lambda_{0}
\end{aligned}
$$

- Modelo 4: Modelo estendido da razão de reatividade [13]

$$
\begin{aligned}
& \frac{d \alpha}{d t}=K_{1}(B+\alpha)(1-\alpha)\left[\lambda_{1}(1-r)+r(1-\alpha)-\lambda_{0}(1-s)\right] \\
& -\frac{d \lambda_{1}}{d t}=2 K_{1}(B+\alpha)(1-\alpha)\left[\lambda_{1}-\lambda_{0}(1-s)\right] \\
& -\frac{d \lambda_{0}}{d t}=4 K_{1}(B+\alpha)(1-\alpha) s \lambda_{0}
\end{aligned}
$$

A constante de velocidade $K_{\mathrm{i}}$ segue a conhecida Lei de Arrhenius e é definida pela Equação (8).

$$
K_{i}=A_{i} e^{\left(-\frac{E_{a}^{i}}{R T}\right)}
$$

em que $A_{i}$ é o fator pré-exponencial, $E_{a}^{i}$ é a energia de ativação, $R$ é a constante universal dos gases, $T$ é a temperatura na qual a reação de cura ocorre e $i$ é o índice igual a 1 ou 2 referente às etapas da reação.

Além das equações do balanço de massa, foram definidas a equação da definição da taxa de aquecimento e a equação para o balanço de energia. Sendo a definição da taxa de aquecimento dada pela Equação 9 .

$$
\frac{d T}{d t}=\Phi
$$

em que $\Phi$ corresponde a taxa de aquecimento em $\mathrm{k} \cdot \mathrm{min}^{-1}$ de cada experimento.

Já a equação do balanço de energia para essa mistura reacional no DSC foi definida pela Equação 10.

$$
m_{e} c_{P} \frac{d T}{d t}=Q+m_{e}(-\Delta H) R_{\alpha}
$$

em que $Q$ é a taxa de calor retirado pelo DSC, $m_{e}$ a massa da amostra utilizada no experimento, $(-\Delta H)$ a entalpia total do sistema, $R_{\alpha}$ a expressão da cinética de cura, $c_{P}$ o calor específico da amostra.

De uma forma geral, os parâmetros dimensionais estimados foram as energias de ativação $E_{l}$ e $E_{2}$ e os fatores pré-exponenciais $A_{1}$ e $A_{2}$ das constantes cinéticas, a razão de reatividade " $r$ ", o parâmetro semiempírico " $s$ ", os expoentes cinéticos $m$ e $n$, além da entalpia de reação $\Delta H$.

\section{RESULTADOS E DISCUSSÃO}

Os modelos foram resolvidos a partir dos dados experimentais que foram cedidos pelo pesquisador Valery Zvetkov, do Institute of Mechanics da Bulgarian Academy of Sciences sediada na cidade de Sofia (Bulgária). Estes dados foram gerados a partir da reação da resina epóxi DGEBA com o mPDA em condições isotérmicas e não isotérmicas por meio do DSC.

Utilizaram-se, para adimensionar o modelo, as relações adimensionais das definições de energia de ativação (11a), fator pré-exponencial (11b), entalpia (11c), tempo (11d) e temperatura (11e). 


$$
\begin{aligned}
\sigma_{i} & =\frac{E_{i}}{R T_{0}} \\
\xi_{i} & =\ln \left(t_{0} A_{i}\right)-\sigma_{i} \\
\eta & =\frac{(-\Delta H)}{c_{p}} \frac{1}{T_{0}} \\
\tau & =\frac{\mathrm{t}}{\mathrm{t}_{0}} \\
\theta & =\frac{\left(T-T_{0}\right)}{T_{0}}
\end{aligned}
$$

em que $T_{0}$ corresponde a uma temperatura de referência, $t_{0}$ um tempo de referência, $\sigma_{i}$ energia de ativação adimensional, $\xi_{i}$ o fator pré-exponencial, $\tau$ o tempo adimensional, $\theta$ a temperatura adimensional.

A partir das definições dessas variáveis adimensionais, as equações na forma adimensional para esse sistema reacional são descritos pela Equação 12 (balanço de energia na forma adimensional), Equação 13 (taxa de aquecimento) e Equação 14 (balanço de massa relacionado com o grau de cura da cinética do modelo).

$$
\begin{aligned}
& \frac{d \theta}{d \tau}=\eta r_{\alpha}+\dot{q} \\
& \frac{d \theta}{d \tau}=\beta \\
& \frac{d \alpha}{d \tau}=r_{\alpha}
\end{aligned}
$$

\begin{tabular}{|c|c|c|c|c|}
\hline Parâmetro & Modelo 1 & Modelo 2 & Modelo 3 & Modelo 4 \\
\hline $\mathrm{E}_{1}\left(\mathrm{~kJ} \mathrm{~mol}^{-1}\right)$ & 71,33 & 79,69 & 71,00 & 70,58 \\
\hline $\mathrm{E}_{2}\left(\mathrm{~kJ} \mathrm{~mol}^{-1}\right)$ & 47,83 & 47,71 & 49,72 & 53,49 \\
\hline$A_{1}\left(s^{-1}\right)$ & $1,53.10^{6}$ & $1,57 \cdot 10^{7}$ & $1,22.10^{6}$ & $4,57.10^{2}$ \\
\hline$A_{2}\left(s^{-1}\right)$ & $9,17.10^{3}$ & $7,50 \cdot 10^{3}$ & $1,82 \cdot 10^{4}$ & 47,83 \\
\hline $\mathbf{m}$ & - & 0,80 & - & - \\
\hline $\mathbf{n}$ & - & 0,92 & - & - \\
\hline $\mathbf{r}$ & - & - & 1,14 & 13101,74 \\
\hline $\mathbf{s}$ & - & - & - & 4189,19 \\
\hline$\Delta \mathbf{H}\left(\mathbf{k J ~ k g}^{-1}\right)$ & $-404,67$ & $-404,55$ & $-411,53$ & $-425,05$ \\
\hline Função objetivo & 4,41 & 3,94 & 4,29 & 7,28 \\
\hline
\end{tabular}

Por meio do ajuste experimental, os valores dos parâmetros adimensionais estimados foram obtidos e estão apresentados na Tabela 1 para o modelo isotérmico. Utilizando-se das relações para adimensionar estes parâmetros, foi possível calcular os parâmetros estimados com suas respectivas unidades para o regime isotérmico, conforme apresentado na Tabela 2.

Tabela 1: Parâmetros adimensionais estimados para os dados isotérmicos.

\begin{tabular}{ccccc}
\hline Parâmetro & Modelo 1 & Modelo 2 & Modelo 3 & Modelo 4 \\
\hline $\boldsymbol{\sigma}_{\mathbf{1}}$ & 24,87 & 27,78 & 24,75 & 24,61 \\
$\boldsymbol{\sigma}_{\mathbf{2}}$ & 16,67 & 16,63 & 17,34 & 18,65 \\
$\boldsymbol{\xi}_{\mathbf{1}}$ & $-2,66$ & $-3,25$ & $-2,78$ & $-12,82$ \\
$\boldsymbol{\xi}_{\mathbf{2}}$ & 0,41 & 0,25 & 0,44 & $-9,12$ \\
$\mathbf{m}$ & - & 0,80 & - & - \\
$\mathbf{n}$ & - & 0,92 & - & - \\
$\mathbf{r}$ & - & - & 1,14 & 13101,70 \\
$\mathbf{S}$ & - & - & - & 4189,19 \\
$\boldsymbol{\eta}$ & 1,17 & 1,17 & 1,19 & 1,23 \\
\hline
\end{tabular}

Tabela 2: Parâmetros dimensionais estimados para os dados isotérmicos.

Procedimento análogo foi realizado para os dados experimentais no regime não isotérmico. $\mathrm{Na}$ Tabela 3 têm-se os parâmetros adimensionais estimados, enquanto a Tabela 4 apresenta os valores dos parâmetros dimensionais com suas respectivas unidades. 
Tabela 3: Parâmetros adimensionais estimados para os dados não isotérmicos.

\begin{tabular}{ccccc}
\hline Parâmetro & Modelo 1 & Modelo 2 & Modelo 3 & Modelo 4 \\
\hline $\boldsymbol{\sigma}_{\mathbf{1}}$ & 18,67 & 13,94 & 19,04 & 17,44 \\
$\boldsymbol{\sigma}_{\mathbf{2}}$ & 17,38 & 22,21 & 16,61 & 18,31 \\
$\boldsymbol{\xi}_{\mathbf{1}}$ & $-3,49$ & $-2,82$ & $-3,32$ & $-5,10$ \\
$\boldsymbol{\xi}_{\mathbf{2}}$ & $-0,19$ & $-1,00$ & $-0,79$ & $-2,06$ \\
$\mathbf{m}$ & - & 0,97 & - & - \\
$\mathbf{n}$ & - & 2,21 & - & - \\
$\mathbf{r}$ & - & - & 5,99 & 5,87 \\
$\mathbf{S}$ & - & - & - & 0,73 \\
$\boldsymbol{\eta}$ & 1,73 & 1,76 & 1,74 & 1,81 \\
\hline
\end{tabular}

Tabela 4: Parâmetros dimensionais estimados para os dados não isotérmicos.

\begin{tabular}{ccccc}
\hline Parâmetro & Modelo 1 & Modelo 2 & Modelo 3 & Modelo 4 \\
\hline $\mathbf{E}_{\mathbf{1}}\left(\mathbf{k J ~ m o l}^{\mathbf{- 1}}\right)$ & 53,56 & 39,97 & 54,60 & 50,02 \\
$\mathbf{E}_{\mathbf{2}}\left(\mathbf{k J ~ m o l}^{-\mathbf{1}}\right)$ & 49,87 & 63,70 & 47,65 & 52,52 \\
$\mathbf{A}_{\mathbf{1}}\left(\mathbf{s}^{-\mathbf{1}}\right)$ & $3,12.10^{3}$ & 53,33 & $5,27.10^{3}$ & $1,80.10^{2}$ \\
$\mathbf{A}_{\mathbf{2}}\left(\mathbf{s}^{-1}\right)$ & $2,30.10^{4}$ & $1,29.10^{6}$ & $5,93.10^{3}$ & $9,01.10^{3}$ \\
$\mathbf{~ m}$ & - & 0,97 & - & - \\
$\mathbf{n}$ & - & 2,21 & - & - \\
$\mathbf{r}$ & - & - & 5,99 & 5,87 \\
$\mathbf{S}$ & - & - & - & 0,73 \\
$\Delta \mathbf{H}\left(\mathbf{k J ~ k g} \mathbf{~ g}^{-1}\right)$ & $-597,98$ & $-608,23$ & $-599,49$ & $-623,90$ \\
Função objetivo & 0,059 & 0,098 & 0,048 & 0,028 \\
\hline
\end{tabular}

Os dados experimentais do fluxo de calor ( $\mathrm{mW}$ ) em função do tempo (min) foram comparados com aqueles preditos pelos modelos estudados. Na Figura 4 tem-se, da esquerda para direita, os Modelos 01, 02, 03 e 04 para os dados isotérmicos, e a Figura 5 apresenta o ajuste dos dados experimentais para o regime não-isotérmico.

Os parâmetros $E_{1}, E_{2}, A_{1}$ e $A_{2}$ encontraram-se, em sua maioria e para os dois regimes, em concordância com aqueles relatados na literatura, sendo a energia de ativação compreendida na

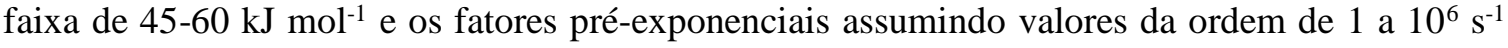
$[7,17]$.

Em relação aos valores da entalpia de reação obtidos, percebe-se boa concordância entre os quatro modelos. No caso da estimação dos dados isotérmicos todos apresentaram resultados dentro da faixa de -400 a $-426 \mathrm{~kJ} \mathrm{~kg}^{-1}$, já os dados não isotérmicos apresentaram valores mais altos e na faixa de -597 a $-624 \mathrm{~kJ} \mathrm{~kg}^{-1}$. Os valores reportados na literatura para sistemas reacionais de resinas epóxi geralmente estão compreendidos na faixa de $-190 \mathrm{~kJ} \mathrm{~kg}^{-1}$ a $-553 \mathrm{~kJ} \mathrm{~kg}^{-1}$ [7,17]. Vale ressaltar que os métodos de estimação dos parâmetros, bem como os endurecedores utilizados junto ao DGEBA na condição não isotérmica afetam os valores de entalpia de reação significativamente. 

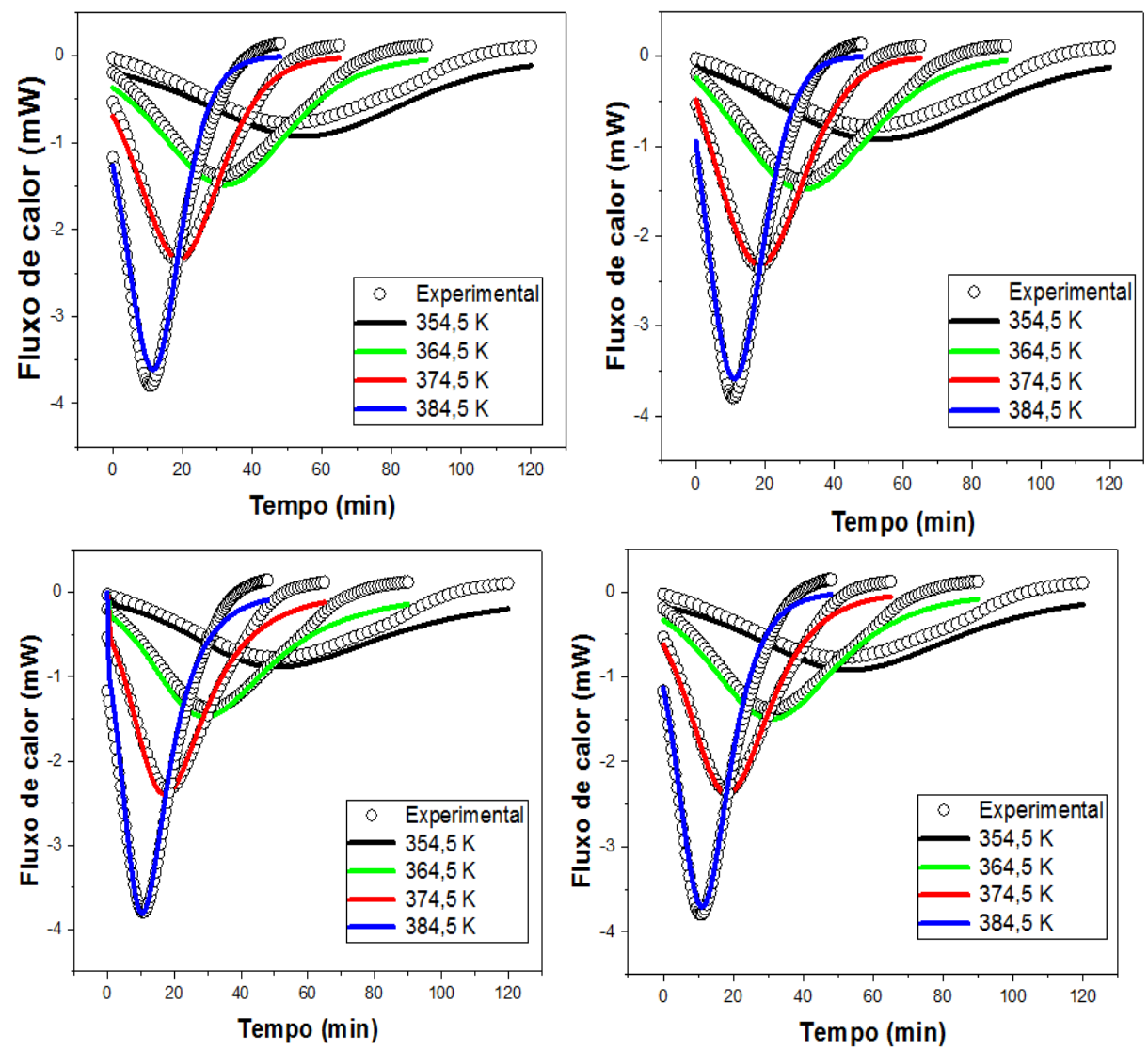

Figura 4: Comparação entre os dados experimentais e os dados preditos dos Modelos 01, 02, 03 e 04 avaliado para o regime isotérmico para a reação do DGEBA com o m-PDA.
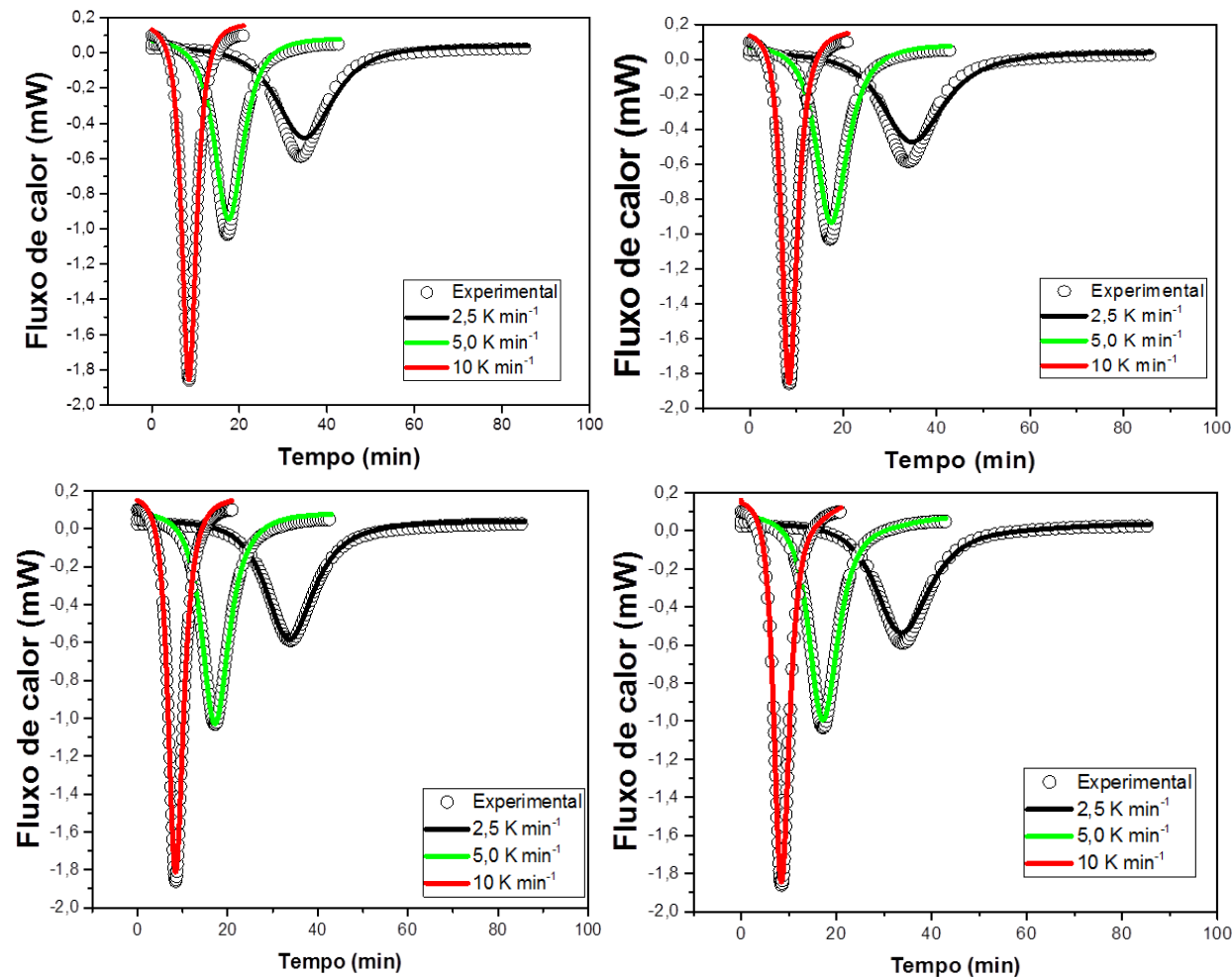

Figura 5: Comparação entre os dados experimentais e os dados preditos dos Modelos 01, 02,03 e 04 avaliado para o regime não-isotérmico para a reação do DGEBA com o m-PDA. 
A partir da análise geral dos parâmetros, em comparação aos valores da literatura e o ajuste de dados, nota-se que o Modelo 01, o Modelo de Horie, mesmo sendo o mais simples, foi o que apresentou resultados mais satisfatórios para a cinética do sistema reacional em situações isotérmicas e não isotérmicas. Comparando-se os Modelos de Horie (01) e Kamal (02), verificase que o modelo de Horie se mostra mais eficiente, mesmo ambos apresentando resultados próximos, devido ao fato do Modelo de Horie exigir menor número de parâmetros. Vale destacar que a diferença principal entre esses modelos está relacionada aos expoentes cinéticos $m$ e $n$ utilizados no Modelo de Kamal. Eles não devem assumir quaisquer valores, sendo que a soma $(m+n)$ define a ordem global da reação da resina epóxi com o agente reticulante e é geralmente dada por, aproximadamente, $(m+n)=2$ ou $(m+n)=3$ [7,17]. As Tabelas 1 e 2 mostram as ordens de reação iguais a 1,77 e 3,18 para os casos isotérmicos e não isotérmicos, respectivamente, em concordância com os valores obtidos na literatura.

No caso dos modelos mecanicistas 03 e 04 , o valor de " $r$ " indica quanto a reatividade dos hidrogênios das aminas secundárias é maior em relação aos hidrogênios das aminas primárias. No Modelo 03, para os dados isotérmicos, a reatividade dos hidrogênios das aminas secundárias foi aproximadamente igual a da amina primária, enquanto que para os dados não isotérmicos os hidrogênios das aminas secundárias apresentaram reatividade seis vezes maior que os hidrogênios das aminas primárias. O Modelo 04, com a adição do parâmetro semiempírico " $s$ " (valor tipicamente menor que um) que busca contabilizar as fracas interações entre as moléculas de amina pura presentes no sistema reacional que podem ser relevantes para a cinética da reação, não apresentou melhora significativa em relação ao Modelo 03 quanto ao ajuste dos dados, podendo-se concluir que o parâmetro " $s$ " não influenciou muito no ajuste. Os valores de " $r$ " e " $s$ " para o Modelo 04 no caso isotérmico apresentaram valores insatisfatórios.

\section{CONCLUSÃO}

Este estudo possibilitou atestar a qualidade dos modelos mecanicistas e empíricos para a representação de dados cinéticos de uma reação de cura por meio da abordagem da solução do sistema algébrico-diferencial utilizando o programa ESTIMA em linguagem FORTRAN.

Os resultados obtidos apresentaram bom ajuste aos dados experimentais, além de apresentarem valores dentro da faixa esperada como descrito na literatura, para essa reação. Apesar dos modelos não apresentarem diferenças significativas no ajuste aos dados, principalmente para o regime não isotérmico, uma comparação com valores da literatura permite observar que o modelo que melhor descreve a reação da cinética da cura do sistema reacional DGEBA - mPDA, em ambos os regimes de processamento da reação, é o Modelo de Horie, sendo o mais simples e se mostrando mais eficiente dentre os propostos.

\section{REFERÊNCIAS BIBLIOGRÁFICAS}

1. Bayraktar E. Introduction. Reference Module in Materials Science and Materials Engineering; 2016.

2. Renda CG. Estudo da resina fenólica fenol/formaldeído e sua aplicação como matriz na preparação de nanocompósitos poliméricos contendo nanotubos de carbono. Dissertação de mestrado, Universidade Federal de Alfenas-MG; 2015. 102 p.

3. Jin FL, Li X, Park SJ. Synthesis and application of epoxy resins: A review. J Ind Eng Chem. 2015;29:111, doi:10.1016/j.jiec.2015.03.026.

4. Ferdosian F, Zhang Y, Yuan Z, Anderson M, Xu C. Curing kinetics and mechanical properties of biobased epoxy composites comprising lignin-based epoxy resins. Eur Polym J. 2016;82:153-165, doi:10.1016/j.eurpolymj.2016.07.014

5. Fache M, Montérémal C, Boutevin B, Caillol S. Amine hardeners and epoxy cross-linker from aromatic renewable sources. Eur Polym J. 2015;73:344-362, doi: 10.1016/j.eurpolymj.2016.07.014.

6. Vidil T, Tournilhac F, Musso S, Robisson A, Leibler L. Control of reactions and network structures of epoxy thermosets. Prog Polym Sci. 2016; doi:10.1016/j.progpolymsci.2016.06.003.

7. Zvetkov VL. Comparative DSC kinetics of the reaction of DGEBA with aromatic diamines: I. Nonisothermal kinetic study of the reaction of DGEBA with m-phenylene diamine. Polymer. 2001;42:66876697, doi:10.1016/S0032-3861(01)00160-4. 
8. Pagano RL, Calado VMA, Tavares FW, Júnior ECB. Cure kinetic parameter estimation of thermosetting resins with isothermal data by using particle swarm optimization. Eur Polym J. 2008;44:2678-2686, doi:10.1016/j.eurpolymj.2008.05.017

9. Cai H, Li P, Sui G, Yu Y, Li G, Yang X, Ryu S. Curing kinetics study of epoxy resin/flexible amine toughness systems by dynamic and isothermal DSC. Thermochim Acta. 2008;473:101-105, doi:10.1016/j.tca.2008.04.012

10. Um MK, Daniel IM, Hwang BS. A study of cure kinetics by the use of dynamics differential scanning calorimetry. Compos Sci Tech. 2009;62:29-40, doi:10.1016/S0266-3538(01)00188-9

11. Zvetkov VL. Comparative DSC kinetics of the reaction of DGEBA with aromatic diamines. II. Isothermal kinetic study of the reaction of DGEBA with mphenylenediamine. Polymer. 2002;43:10691080, doi:10.1016/S0032-3861(01)00695-4

12. Horie K, Hiura H, Sawada M, Mita I, Kambe H. Calorimetric investigation of polymerization reactions. III. Curing reaction of epoxides with amines. J Polym Sci. 1970;A8:1357, doi:10.1002/pol.1970.150080605

13. Zvetkov VL. Mechanistic modeling of the epoxy-amine reaction. Model derivations. Thermochim Acta. 2005;435:71-84, doi:10.1016/j.tca.2005.04.025

14. Ren R, Xiong X, Ma X, Liu S, Wang J, Chen P, Zeng Y. Isothermal curing kinetics and mechanism of DGEBA epoxy resin with phthalide-containing aromatic diamine. Thermochim Acta. 2016;623:15-21, doi:10.1016/j.tca.2015.11.011

15. Zvetkov VL, Simeonva-Ivanova E, Calado V. Comparative DSC kinetics of the reaction of DGEBA with aromatic diamines. IV. Iso-conversional kinetic analysis. Thermochim Acta. 2014;596:42-48, doi:10.1016/j.tca.2014.09.014

16. Kamal MR, Sourour S, Ryan M. Integrated thermo-rheological analysis of the cure of thermosets. SPE Tech Paper. 1973;19:187-191

17. Santos DC, Jesus LFS, Santana PL, Calado MVA, Biscaia EC, Pagano RL. Determinação de parâmetros cinéticos da reação de cura de resinas poliméricas utilizando modelos empíricos e mecanicistas. Sci Plena. 2016;12(05):1-10, doi:10.14808/sci.plena.2016.054215

18. Silva CL, Santana PL, Silva CF, Pagano RL. Modelagem e estimação de parâmetros do processo de produção de etanol em reator batelada por Saccharomyces cerevisiae. Sci Plena. 2016;12(05):1-14, doi:10.14808/sci.plena.2016.054216 